\title{
Tissue inhibitor of metalloproteinases-1 protects human neurons from staurosporine and HIV-1-induced apoptosis: mechanisms and relevance to HIV-1-associated dementia
}

\author{
Ashutosh $^{1,3}$, C Chao ${ }^{1,3}, K_{\text {Borgmann }}{ }^{1}, \mathrm{~K} \mathrm{Brew}^{2}$ and A Ghorpade ${ }^{\star, 1}$
}

HIV-1-associated dementia (HAD)-relevant proinflammatory cytokines robustly induce astrocyte tissue inhibitor of metalloproteinases-1 (TIMP-1). As TIMP-1 displays pleotropic functions, we hypothesized that TIMP-1 expression may serve as a neuroprotective response of astrocytes. Previously, we reported that chronically activated astrocytes fail to maintain elevated TIMP-1 expression, and TIMP-1 levels are lower in the brain of HAD patients; a phenomenon that may contribute to central nervous system pathogenesis. Further, the role of TIMP-1 as a neurotrophic factor is incompletely understood. In this study, we report that staurosporine (STS) and HIV-1 $1_{\mathrm{ADA}}$ virus, both led to induction of apoptosis in cultured primary human neurons. Interestingly, cotreatment with TIMP-1 protects neurons from apoptosis and reverses neuronal morphological changes induced by these toxins. Further, the anti-apoptotic effect was not observed with TIMP-2 or -3 , but was retained in a mutant of the $\mathrm{N}$-terminal TIMP-1 protein with threonine-2 mutated to glycine (T2G) that is deficient in matrix metalloproteinase (MMP)-1, -2 and -3 inhibitory activity. Therefore, the mechanism is specific to TIMP-1 and partially independent of MMP-inhibition. Additionally, TIMP-1 modulates the Bcl-2 family of proteins and inhibits opening of mitochondrial permeability transition pores induced by HIV-1 or STS. Together, these findings describe a novel function, mechanism and direct role of TIMP-1 in neuroprotection, suggesting its therapeutic potential in HAD and possibly in other neurodegenerative diseases.

Cell Death and Disease (2012) 3, e332; doi:10.1038/cddis.2012.54; published online 28 June 2012

Subject Category: Neuroscience

The tissue inhibitors of metalloproteinases (TIMP) family are composed of four members (TIMP-1, 2, 3, and 4), which, by definition, inhibit the activity of matrix metalloproteinases (MMP), a large family of zinc-dependent proteases. The TIMP/MMP balance is crucial in extracellular matrix (ECM) homeostasis and remodeling; which is essential for diverse physiological processes including cell growth, migration and apoptosis in a variety of cells. ${ }^{1}$ The MMP/TIMP imbalance is mechanistically implicated in several central nervous system (CNS) and neurodegenerative diseases, including ischemia, Alzheimer's disease and neuroinflammatory diseases like human immunodeficiency virus (HIV)-1-associated dementia (HAD). ${ }^{2}$ As each TIMP can inhibit a wide spectrum of MMPs, TIMPs affect many cellular functions such as growth, migration, and apoptosis. However, it is now strongly evident by the identification of specific binding partners on cells that these cellular activities of TIMPs may or may not require MMP-inhibitory activity. ${ }^{1}$

In the CNS, TIMP-1 expression increases significantly during postnatal development as compared with adult, but TIMP-1 is currently studied with particular interest in disease process because of its robust overexpression in response to inflammatory myelin injury. ${ }^{3}$ As TIMP-1 is predominantly expressed by astrocytes surrounding white matter lesions, it likely acts as an endogenous factor to rescue cells from the toxic effects of MMP activities during neuroinflammation. ${ }^{4}$ However, besides the classical function of MMP-inhibition, TIMP-1 also possesses growth factor activity and antiapoptotic potential; which may be mediated through ligandreceptor interactions. ${ }^{5-7}$ Hence, TIMP-1 biological functions are not limited to MMP-inhibition, but may include development, CNS injury, and inflammation. ${ }^{8}$

\footnotetext{
${ }^{1}$ Department of Cell Biology and Anatomy, University of North Texas Health Science Center, Fort Worth, TX 76107, USA and ${ }^{2}$ Department of Biomedical Sciences, Florida Atlantic University, Boca Raton, FL 33431, USA

*Corresponding author: A Ghorpade, Department of Cell Biology and Anatomy, University of North Texas Health Science Center, 3500 Camp Bowie Boulevard, RES 202, Fort Worth, TX 76107, USA. Tel: 817735 2048; Fax: 817735 2610; E-mail: Anuja.Ghorpade @ unthsc.edu.

${ }^{3}$ These authors contributed equally to this work.

Keywords: tissue inhibitor of metalloproteinases-1; staurosporine; neurotoxicity; neuroprotection; apoptosis; HIV-1-associated dementia

Abbreviations: BSA, bovine serum albumin; BDNF, brain-derived neurotrophic factor; Calcein-AM, acetomethoxy derivate of calcein; CNS, central nervous system; $\mathrm{CoCl}_{2}$, cobalt chloride; DAPI, 4',6-diamidino-2-phenylindole; dsDNA ELISA, double-stranded DNA fragmentation enzyme-linked immunosorbent assay; EAE, experimental autoimmune encephalomyelitis; ECM, extracellular matrix; GFAP, glial fibrillary acidic protein; HAD, HIV-1-associated dementia; HBSS, Hank's-buffered salt solution; HIV-1, human immunodeficiency virus-1; IL, interleukin; MAP, microtubule-associated protein; MMP, matrix metalloproteinases; mPTP, mitochondrial permeability transition pore; MTT, 3-(4,5-dimethylthiazol-2-yl)-2,5-diphenyltetrazolium bromide; PI3K, phosphotidylinositol-3 kinase; S.E.M., standard error of mean; STS, staurosporine; T2G, N-terminal TIMP-1 protein with threonine-2 mutated to glycine; TIMP, tissue inhibitors of metalloproteinases; TUNEL, terminal deoxynucleotidyltransferase-mediated dUTP-nick end labeling

Received 27.1.12; revised 27.3.12; accepted 10.4.12; Edited by A Verkhratsky
} 
We and other laboratories have previously reported that astrocytes increase the production of TIMP-1 in response to proinflammatory cytokines, such as interleukin (IL)-1 $\beta$ and/or tumor necrosis factor $\alpha$ indicating a role of TIMP-1 in neuroinflammation., ${ }^{8,9}$ Astrocytic TIMP-1 expression in response to pro-inflammatory cytokines is substantial because astrocytes, not microglia, upregulate TIMP-1 levels upon activation with IL-1 $\beta .{ }^{10}$ Previously, we have shown that, in contrast to acute inflammation, TIMP-1 levels decrease during chronic inflammation in vitro, as well as in cerebrospinal fluid (CSF) and brain tissue of HAD patients. ${ }^{9}$ These data indicate that the initial robust injury-induced astrocyte TIMP-1 expression may serve to protect from neuronal damage at the inflammatory foci. Interestingly, TIMP-1 inhibits excitotoxic cell death in neuronal cells in animal models, likely through the modulation of intracellular calcium levels, but specific mechanistic studies in context of human targets and HAD are lacking. ${ }^{11}$ The analysis of brain tissues from HADpatients, animal and cell culture models of the disease strongly indicated that apoptosis was a predominant contributor to neuronal death in HIV-1 CNS infection. ${ }^{12}$ In these studies, neuronal apoptosis was characterized morphologically by decrease in cell volume, bleb-like cell surface protuberances, chromatin condensation and DNA fragmentation. However, the exact mechanism(s) of HIV-1-induced neuronal apoptosis remains unknown. Understanding the mechanisms operating in the CNS to protect neurons from toxicity by HIV-1 is critical from a clinical perspective and for developing rational therapies that can prevent neuronal death after injury or long-term disease. These factors led us to hypothesize that TIMP-1 may have a neuroprotective role in CNS during HAD. There are multiple pathways and mechanisms that may be responsible for HIV-1-induced apoptosis in neurons; therefore, a broad-spectrum apoptosis-inducing agent should be used in order to study TIMP-1 neuroprotective effects.

Herein, we report the neuroprotective potential of TIMP-1 in cultured primary human neurons against HIV-1 and staurosporine (STS), a model cytotoxin extensively used to decipher the underlying mechanisms of apoptosis. ${ }^{13}$ Our results demonstrate that TIMP-1-mediated neuroprotection is MMPindependent, at least in part, through modulation of $\mathrm{Bcl}-2$ family of proteins, decreasing of the opening of mitochondrial membrane permeability transition pore (mPTP) and eventually preventing DNA fragmentation. Results from this study identify TIMP-1 as a novel astrocyte-derived factor that can directly influence neuronal survival during HIV-1-associated neurotoxicity.

\section{Results}

TIMP-1 protects human neurons against STS-induced toxicity. We first examined the effect of TIMP-1 on human neuronal survival during STS-induction of apoptosis. STStreatment significantly increased the percentage of terminal deoxynucleotidyltransferase-mediated dUTP-nick end labeling (TUNEL)-positive neuronal nuclei ( $35 \%, P<0.001$; Figures 1d and $\mathrm{g}$ ), which was significantly reduced upon TIMP-1 cotreatment ( $~ 50 \%, P<0.001$; Figures $1 \mathrm{e}$ and $\mathrm{g}$ ).
As previously reported, ${ }^{14}$ brain-derived neurotrophic factor (BDNF) also significantly reduced percent apoptotic nuclei in STS-challenged neurons $(P<0.001$; Figures $1 \mathrm{f}$ and $\mathrm{g})$. Moreover, TIMP-1-treatment alone improved the morphology of neurons (Figures $1 \mathrm{~b}$ and $\mathrm{g}$ ) and decreased basal apoptosis significantly as compared with untreated control $(P<0.001$; Figures $1 \mathrm{~g}$ and $\mathrm{i})$. This could be due to an innate neurotrophic activity of TIMP-1. Cotreatment of the neurons with STS and either TIMP-1 or BDNF significantly improved the metabolic activity in neurons (Figure $1 \mathrm{~h} ; P<0.01$ and $P<0.05$, respectively). STS + TIMP-1 and STS + BDNF groups were not statistically different, indicating that TIMP-1 is comparable to BDNF in its ability to protect neurons against STS-induced neurotoxicity. In parallel experiments, we determined TIMP-1 neuroprotection by quantitatively measuring double-strand DNA fragments by double-stranded DNA fragmentation enzyme-linked immunosorbant assay (dsDNA ELISA). STS induced a dose-dependent increase in DNA fragmentation in human neurons $(P<0.001$, Figure 1i), which was decreased significantly upon TIMP-1-treatment as compared with the same dose of STS-alone $(P<0.001$, Figure 1i). These observations suggest that TIMP-1 is a pro-survival and anti-apoptotic factor for neurons and contributes to the suppression of apoptosis induced by STS in human neurons.

Neuroprotection is specific to TIMP-1 and likely independent of MMP inhibition. As TIMPs have divergent effects on proliferation and apoptosis independent of their MMP-activity in a number of cell types, we next investigated if TIMP-1 protects neurons because of its intrinsic property of MMP-inhibition. ${ }^{1}$ By using a N-terminal TIMP-1 protein with threonine-2 mutated to glycine (T2G) which differs from the wild-type protein by a single-amino-acid substitution, and reduces the inhibition constant of TIMP-1 for MMP-1 and MMP-3 by a factor of over 1000 and an $\sim 20$-fold effect on MMP-9. ${ }^{15,16}$ Moreover, the secondary structure of this mutant protein is not significantly different than wild type. Therefore, any neuroprotective effect of T2G treatment is MMP-independent. ${ }^{17}$

Neuronal cultures were treated with STS and the morphological changes were visualized by immunocytochemical staining for microtubule-associated protein (MAP)-2, neuron specific marker. The characteristic morphological changes due to apoptosis were observed in STS-challenged neurons, viz. significant loss of 4',6-diamidino-2-phenylindole (DAPI)stained nuclei and MAP-2-positive cells (Figure 2b). Furthermore, shrunken cell bodies with beading, fragmented, or obliterated neuronal processes were observed in remaining STS-treated MAP-2-positive neurons confirming the cytotoxic effects of STS on neuronal morphology and survival. Cotreatment of neurons with STS and either TIMP-1, T2G mutant or BDNF considerably prevented STS-induced cell death compared with STS alone, and STS in combination with BSA (bovine serum albumin, negative control) (Figures 2a and $c-f)$. Moreover, cotreatment with TIMP-1, T2G and BDNF also prevented impairments in morphology, integrity of cell body, as well as neurite network and processes. Furthermore, neuroprotection by the T2G mutant was 

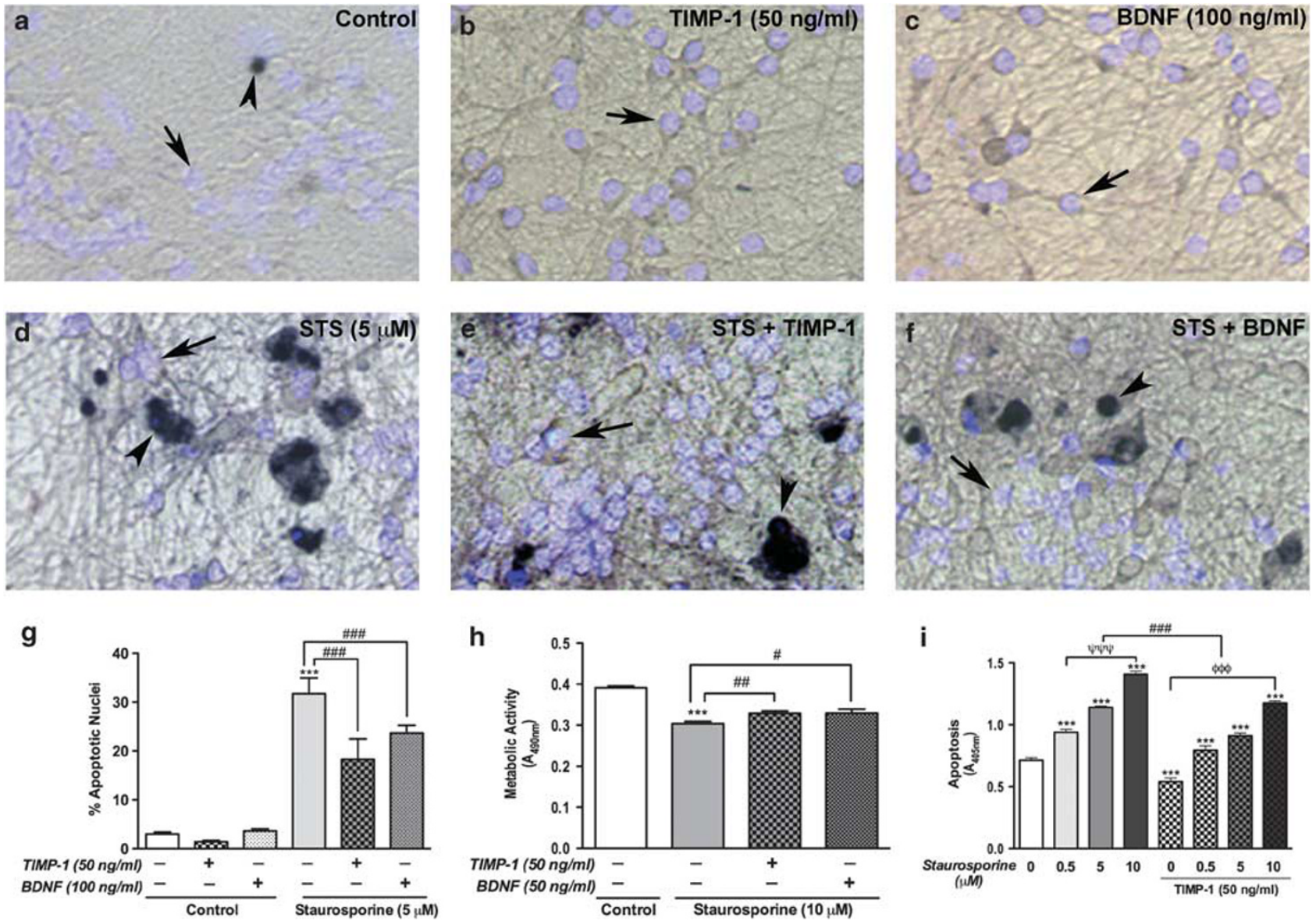

Figure 1 TIMP-1 enhances neuronal survival against STS-cytotoxicity. Primary human neurons were treated with STS with or without $50 \mathrm{ng} / \mathrm{ml}$ of TIMP-1 or BDNF for $24 \mathrm{~h}$. The cells were fixed and stained (a-f) with TUNEL and DAPI (nuclei with fragmented DNA stained black, arrowhead; while nuclei with intact DNA stained blue, arrow). Percentage of apoptotic nuclei in all cells counted in a total of eighteen random microscope fields from three replicates were calculated (g). A dose-dependent effect of STS and TIMP-1-mediated neuroprotection was measured by cell viability assay $(\mathbf{h})$ and quantitative analysis of apoptosis by dsDNA ELISA (i). Data representative of at least three independent biological replicates are shown and presented as mean \pm S.E.M. Symbols centered over the error bar indicate the relative level of the significance compared with untreated control ( $\left.{ }^{\star \star \star} P<0.001\right)$, STS-alone $\left({ }^{\sharp} P<0.05\right.$, ${ }^{\# \#} P<0.01$ and $\left.{ }^{\# \# \#} P<0.001\right)$ or comparisons between TIMP-1 doses $\left({ }^{\phi \phi \phi} P<0.001\right)$ or between STS doses $\left({ }^{\psi} \psi \psi P<0.001\right)$

comparable to wild type TIMP-1 or BDNF as assayed by 3-(4,5-dimethylthiazol-2-yl)-2,5-diphenyltetrazolium bromide (MTT) assay as a measure of metabolic activity (Figure $2 \mathrm{~g}$ ). Based upon these studies, TIMP-1-mediated neuroprotection appears to be independent of its MMP-inhibition activity.

As, the TIMP family members exhibit promiscuity in their MMP-inhibition activity, we determined whether the neuroprotective functions of TIMP-1 are shared by TIMP-2 and -3, each of which binds and inhibits MMP activity. TIMP-2 binds pro-MMP-9, and was specifically included to determine if MMP-9 inhibition has a role in TIMP-1 neuroprotection, as the T2G mutant had reduced but continued MMP-9 inhibiting capacity. As observed by the above-mentioned assays, STS significantly increased the neuronal death as measured by trypan-blue exclusion assay (Figures $2 \mathrm{~b}$ and $\mathrm{g} ; P<0.001$ ). TIMP-1 and T2G mutant both significantly decreased the percentage of apoptotic cells (Figures 3c, d, and g; $P<0.01$, respectively). However, TIMP-2 and TIMP-3, which have MMP-inhibiting activities, did not display significant neuroprotective effects as compared with STS-alone (Figures $3 e-g ; P>0.05)$. Thus, these results strongly suggest that TIMP-1-mediated neuroprotection is specific and MMPindependent.

TIMP-1 mediates neuroprotection against HIV-1 $\mathbf{1}_{\text {ADA }}$ virus. We next evaluated the relevance of neuroprotective potential of TIMP-1 in HAD by treating neurons with macrophage-tropic HIV-1 $1_{A D A}$ viral particles, with or without TIMP-1 cotreatment. TIMP-1 ameliorated the degradation in neuronal morphology, shrinkage of cell bodies and fragmentation of neuronal processes exerted by HIV-1 $1_{\text {ADA }}$ on human neurons (Figures $4 \mathrm{c}$ and d). TIMP-1-cotreatment significantly reduced apoptosis ( $\sim 20 \%, P<0.001)$ as compared with HIV-1 $1_{\mathrm{ADA}}$-alone neurons $(\sim 80 \%$, Figures $4 \mathrm{C}$ and $e$; $P<0.001)$ as determined by TUNEL-assay. Taken together, these results confirmed TIMP-1 neuroprotection as evident by decreased apoptosis and preservation of cellular morphology in the presence of HAD-specific neurotoxin, HIV $-1_{\text {ADA. }}$.

TIMP-1 inhibits MPTP opening. The opening of MPTP is an early apoptotic event in neurons and represents the "point 

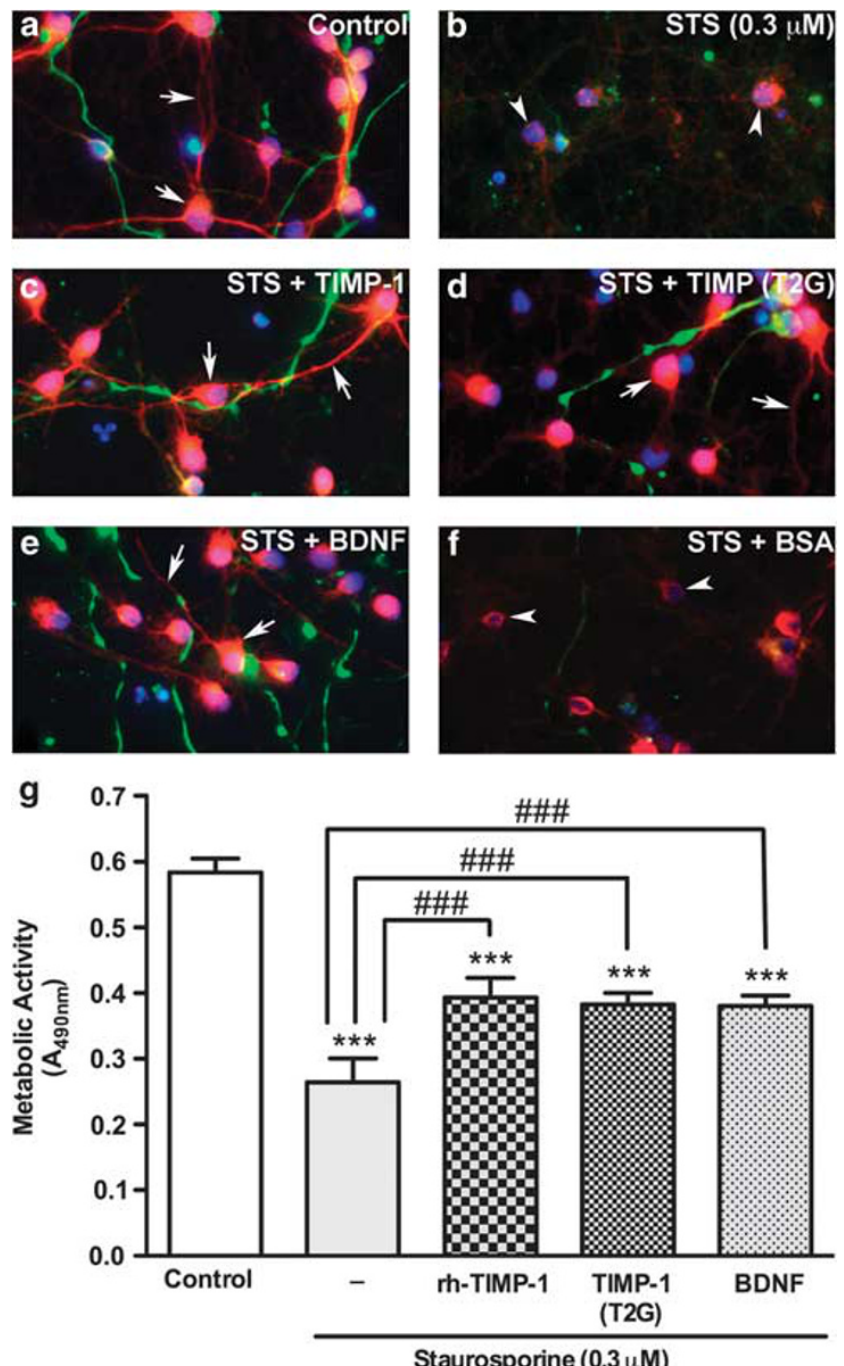

Figure 2 TIMP-1 prevents the morphological and metabolic changes induced by STS. Human neurons challenged with STS $(0.3 \mu \mathrm{M})$ for $24 \mathrm{~h}$, with or without $50 \mathrm{ng} / \mathrm{ml} \mathrm{BDNF}$, TIMP-1, TIMP-2 or $28 \mathrm{ng} / \mathrm{ml}$ T2G mutant were assessed for morphological changes and/or preservation/protection by immunocytochemistry (a-f) and cell viability by MTT assay (g). Representative micrographs of immunofluorescent staining (red: MAP-2, neuronal marker, specific cytoskeletal proteins that are enriched in dendrites and essential to stabilize its shape; green: GFAP for astrocytes; blue: DAPI for nuclei). Arrows represent the MAP-2 enriched neurons with DAPI and processes, while arrowheads represent loss of MAP-2, DAPI and processes. Images are representative of five random fields (original magnification $\times 200$ ) in each replicate, three replicates per condition. Data represents at least three independent biological replicates and presented as mean \pm S.E.M. Symbols centered over the error bar indicate the relative level of the significance compared with untreated control $\left({ }^{* \star *} P<0.001\right)$ or STS-alone $\left({ }^{\# \#} P<0.001\right)$

of no return' of the lethal process. ${ }^{18}$ As TIMP-1 protects human neurons from STS and HIV-1 $1_{\text {ADA }}$ insult in an MMPindependent manner, we studied whether TIMP-1 can inhibit mPTP opening in HIV-1 or toxin challenged neurons by acetomethoxy derivate of calcein (calcein-AM) and cobalt chloride $\left(\mathrm{CoCl}_{2}\right)$ assay. Calcein (green) colocalization in mitochondria with MitoTraker (Life Technologies, Carlsbad, CA, USA) (red) is visualized as a yellow fluorescence. The green/yellow fluorescence is lost upon calcein release from
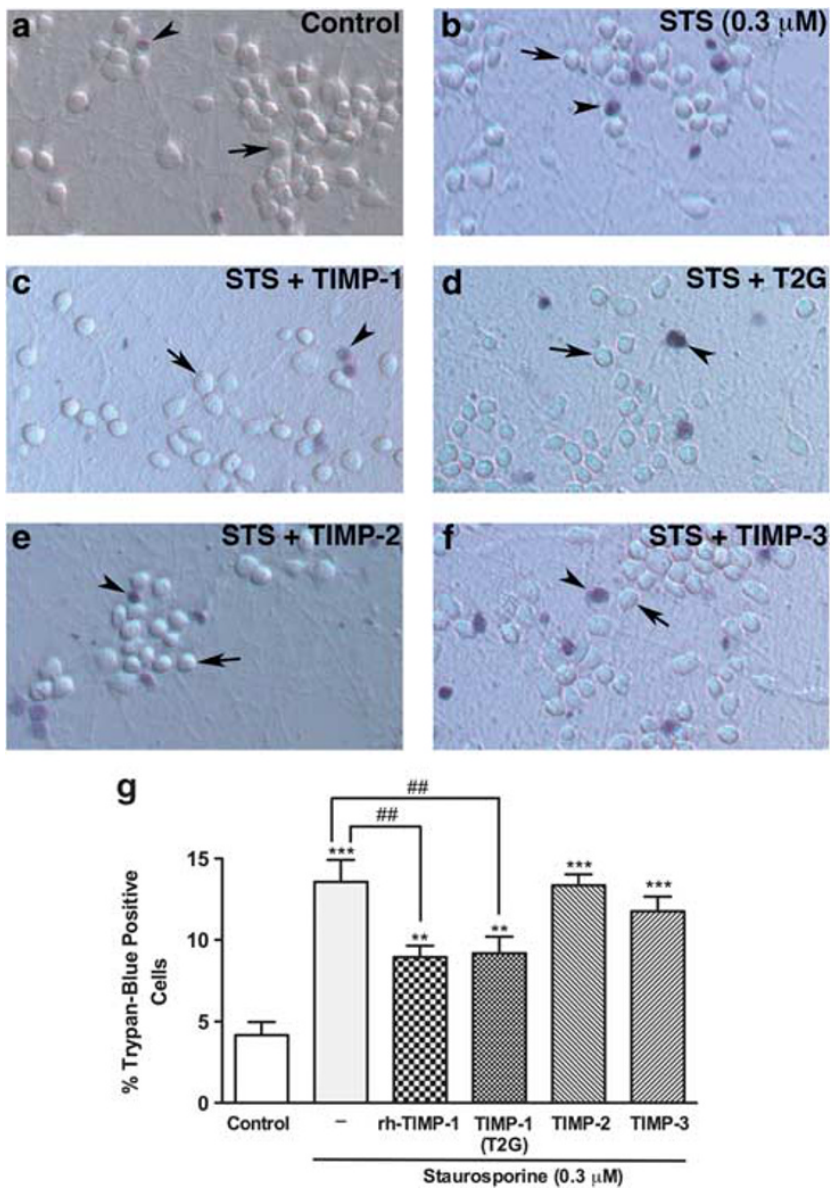

Figure 3 TIMP-1-mediated neuroprotection is specific to TIMP-1 and MMPindependent. The cell viability assay was performed in human neurons after exposure of STS $(0.3 \mu \mathrm{M})$ for $18 \mathrm{~h}$ with or without $50 \mathrm{ng} / \mathrm{ml}$ of TIMP-1, $-2,-3$ or $28 \mathrm{ng} / \mathrm{ml} \mathrm{T2G}$. Panels are representative micrographs of 10 random fields (original magnification $\times 200$ ) from two replicates of control $(a)$, STS $(b)$ and STS with TIMP-1 (c), T2G (d), TIMP-2 (e) or TIMP-3 (f). Trypan-blue positive cells were considered as dead cell (represented by arrowhead) while, cells that excluded trypan-blue were counted as live (represented by arrow) and percentage of trypanblue positive cells was calculated $(\mathbf{g})$. Data is representative of at least three independent biological replicates and presented as mean \pm S.E.M. Symbols centered over the error bar indicate the relative level of the significance compared with untreated control ( ${ }^{* \star} P<0.01$ and $\left.{ }^{* \star *} P<0.001\right)$ or STS-alone $\left({ }^{\# \#} P<0.01\right)$

mitochondria consequent to MPTP opening and subsequent quenching of fluorescence by cobalt in cytoplasm. HIV-1 ADA viral particles or STS induced opening of MPTP in neurons as depicted by loss of green/yellow fluorescence (Figure 5). However, cotreatment of TIMP-1 inhibited MPTP opening as observed by the preservation of green/yellow fluorescence baseline levels. Moreover, HIV-1 ${ }_{\text {ADA- }}$ or STS-treatment alone significantly decreased the number of live, green fluorescent cells (as detected only by nuclear stain), as only live cells can take up calcein-AM and metabolize it into the green fluorescent product (calcein). TIMP-1 cotreatment preserved the number of green fluorescent cells, indicating overall cellular protection in neurons. Together, the data demonstrate that the improved cell viability and decreased apoptosis mediated by TIMP-1 were accompanied by inhibition of MPTP opening. 

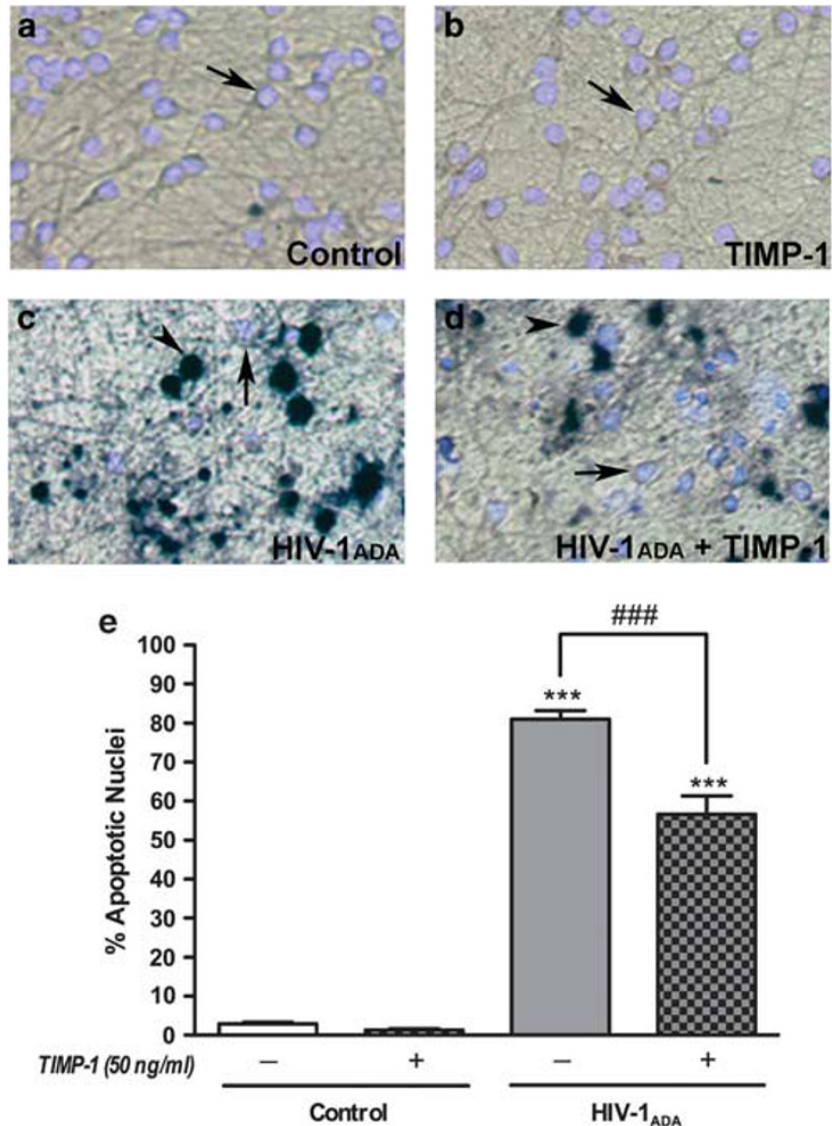

Figure 4 TIMP-1 mediates neuroprotection against HAD-relevant HIV-1 virus. Human neurons were fixed and assayed with TUNEL assay after treatment with clarified HIV-1 $1_{\text {ADA }}$ and $/$ or $50 \mathrm{ng} / \mathrm{ml}$ TIMP-1 for $20 \mathrm{~h}$. Panels are representative micrograph (original magnification X200) overlays of TUNEL and DAPI staining from control (a), TIMP-1 (b), HIV-1 ADA $_{\text {(c) and HIV-1 }}$ ADA + TIMP-1 (d). Arrows and arrowheads represent TUNEL-positive or negative cells, respectively. Cell counts from five random microscope fields in each replicate, three replicates per condition were analyzed as \% apoptotic (TUNEL-positive) cells (e). Data is representative of at least three independent biological replicates and presented as mean \pm S.E.M. Symbols centered over the error bar indicate the relative level of the significance

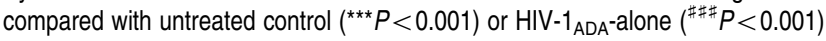

TIMP-1 modulates Bcl-2 family of proteins for neuroprotection. The fate of the cell is determined in a large measure by the balance of pro-apoptotic and anti-apoptotic proteins; therefore, we studied the effect of TIMP-1 on modulation of $\mathrm{Bcl}-2$ family of proteins. The Bcl-2 family of proteins, including $\mathrm{Bcl}-2, \mathrm{Bcl}-\mathrm{x}_{\mathrm{L}}$ and $\mathrm{Bax}$ are pivotal regulators of intrinsic apoptotic signaling and regulate MPTP opening. $\mathrm{Bcl}-2$ and $\mathrm{Bcl}-\mathrm{x}_{\mathrm{L}}$ exert their effects by contributing to the maintenance of mitochondrial membrane integrity. In contrast, Bax compromises membrane integrity leading to MPTP opening and leakage of apoptotic factors, such as cytochrome $c$ and apoptosis-inducing factor. ${ }^{19,20}$ A robust increase in Bax (2.84 \pm 0.02 -fold) and decrease Bcl-2 and $\mathrm{Bcl}-\mathrm{x}_{\mathrm{L}}$ levels (approximately two-fold) were observed by STS-treatment (Figure 6), consistent with studies performed on rat neurons and neuroblastoma cells. ${ }^{13,21}$ STS dramatically increased the ratios of $\mathrm{Bax} / \mathrm{Bcl}-2$ and $\mathrm{Bax} / \mathrm{Bcl}-\mathrm{x}_{\mathrm{L}}$ by $6.22 \pm 0.12-$ and $6.25 \pm 0.28$-fold, respectively. Interestingly, both TIMP-1 and T2G mutant substantially inhibited STSinduced changes in the expression of $\mathrm{Bcl}-2$ and $\mathrm{BCl}-\mathrm{x}_{\mathrm{L}}$ and Bax, preserving the ratios of $\mathrm{Bax} / \mathrm{Bcl}-2$ and $\mathrm{Bax} / \mathrm{Bcl}-\mathrm{x}_{\mathrm{L}}$ levels at basal control levels (Figures $6 \mathrm{a}-\mathrm{c}$ ). A direct indication of MMP-independent neuroprotection by TIMP-1 was observed as there was no detectable difference between the expression of these proteins in TIMP-1 and T2G mutant treated cells; however, TIMP-2 did not show this effect. As expected, BDNF increased the expression of Bcl-2, Bcl- $x_{L}$ and decreased the expression of Bax and also preserved the basal control ratios. ${ }^{22}$ Taken together, these results suggest TIMP-1 increases the expression of anti-apoptotic proteins in neurons challenged with cytotoxic stimuli and also inhibits the expression of pro-apoptotic proteins, which protects neurons from mPTP opening and shifts the fate of neurons from apoptosis toward pro-survival pathways. Further, this activity is TIMP-1 specific and mediated by regulation of mPTP opening, independent of MMP-inhibition, suggesting a direct effect modulating the cell fate by regulating initiation of apoptosis.

\section{Discussion}

Neuroinflammation induced because of chronic HIV-1 infection in the CNS is an intense topic of investigation in order to both expand the mechanistic framework of the underlying pathophysiology and to characterize potential targets for further therapeutic interventions. In this regard, the current investigations have characterized that the initial TIMP-1 upregulation in reactive astrocytes during HIV-1 CNS infection is a protective mechanism against HIV-1-associated neurotoxicity. Thus, loss of TIMP-1 during chronic inflammation may not only exacerbate ECM breakdown due to lack of MMP inhibition, but also lead to loss of direct neuroprotective effects mediated by TIMP-1.

Our studies, for the first time, identified the neuroprotective potential of TIMP-1 in cultured primary human neurons from toxicity induced by both HIV-1 $1_{\text {ADA }}$ and cytotoxins such as STS. Our investigations demonstrated that the anti-apoptotic property of TIMP-1 is direct, specific and independent of its MMP-inhibition abilities. The exogenous supply of recombinant human TIMP-1 not only increased the cell viability, but also preserved the cell morphology following cytotoxic

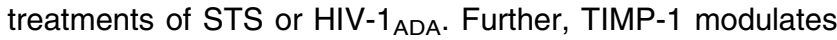
the expression of $\mathrm{Bcl}-2$ family of proteins and inhibits $\mathrm{MPTP}$ opening, which promote neuronal cell survival pathways. It is noteworthy that neuroprotective effect of TIMP-1, T2G mutant or BDNF was not only quantitatively but also mechanistically comparable in the studied parameters.

The MMP activity is tightly regulated through gene expression and protein secretion, proenzyme activation, sequestration and inhibition by their endogenous inhibitors, TIMPs. ${ }^{23} \mathrm{An}$ TIMP/MMP imbalance and ECM degradation are implicated in a variety of neuroinflammatory diseases including HAD. ${ }^{8}$ Together, TIMP-1 and -2 possess the potential to inhibit the activities of all known MMPs (although TIMP-1 is a poor inhibitor of MMP-14, 16, -19 and -24) and have an essential role in maintaining the balance between ECM formation and degradation. ${ }^{8}$ Interestingly, TIMP-1 and -2 levels increase in many neurodegenerative disorders, including Alzheimer's 

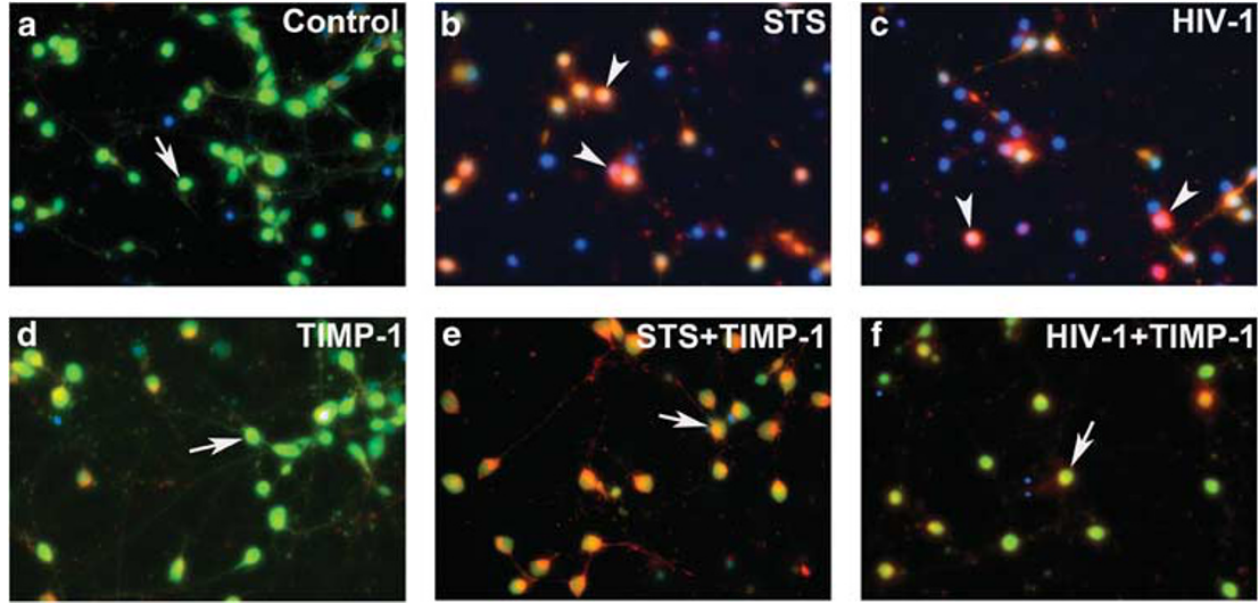

Figure 5 The TIMP-1 inhibits the mPTP opening induced by HIV-1 ${ }_{\mathrm{ADA}}$ or STS. The changes in mPTP in human neurons were monitored by Calcein/CoCl 2 assay. Calcein (green fluorescence) colocalized with MitoTraker (red) represents closed mPTP (arrows), while loss of mitochondrial green fluorescence represents opening of mPTP (arrowheads). Control (a) and TIMP-1 treated neurons (d) show bright calcein fluorescence (green/yellow) indicating intact mitochondria. Neurons treated with STS or HIV-1 display a significant decrease in calcein fluorescence suggest opening of mPTP and quenching of calcein fluorescence by cytoplasmic $\mathrm{CoCl}_{2}$ (b and $\mathbf{c}$ ). Simultaneous treatment of TIMP-1 significantly blocked the decrease in calcein fluorescence caused by STS or HIV-1 suggesting it inhibits opening of mPTP (e and f)

disease, Huntington's disease, Parkinson's disease, and amyotrophic lateral sclerosis. ${ }^{8}$ We have previously reported that TIMP-1 is inducible in astrocytes after acute exposure to IL-1 $\beta .^{9}$ Further, TIMP-1 protein levels decrease and MMP-2 and pro-MMP-9 levels increase in the CSF of HAD patients as compared with non-demented HIV-1 seropositive or seronegative controls. ${ }^{9}$ The differential regulation of TIMP-1 by glia during neuroinflammation serves multiple purposes. For example, increased MMP expression can facilitate MP infiltration into the CNS in HAD involving degradation of the ECM, which can be countered by TIMP-1. ${ }^{24}$ Owing to MMPinhibition activity, TIMP-1 has a variety of roles, viz. protecting neurons during severe cerebral ischemia, ${ }^{25}$ reducing neurite length, increasing the size of growth cones, ${ }^{26}$ generation and differentiation of oligodendrocytes ${ }^{27}$ and attenuation of demyelination in experimental autoimmune encephalomyelitis (EAE). ${ }^{4}$

The adenoviral delivery of TIMP-2 also had a protective effect in cerebral ischemia due to MMP-inhibition activity. ${ }^{28}$ TIMP-1 protects rat hippocampal neuronal death in culture against glutamate-induced excitotoxicity. ${ }^{11}$ Our results show the neuroprotective potential of TIMP-1 and demonstrate its role in protection from HIV-1-induced neurotoxicity for the first time. Additionally, TIMP-1 also protected human neurons from a broad-spectrum cytotoxin, STS. There are indications that TIMP-1 is implicated in diverse neuroprotection pathways during various neuronal injuries, viz. inflammatory, (EAE model), ${ }^{4}$ excitoxicity (glutamate dysregulation), ${ }^{11}$ and neuronal activity-dependent injuries (kainate-seizure mouse model). ${ }^{5}$ TIMP-1 has also been linked to ECM preservation through MMP-inhibition, non-inhibiting MMP-regulation and through the non-classical modulation of ionotropic glutamate receptors. ${ }^{5,11,29}$ Studies with potassium cyanide and TIMP-1 knockout also indicate that TIMP-1-mediated neuroprotection may not be evident in all types of neuronal injuries. ${ }^{5,11}$ Thus, TIMP-1 has differential effects on neurons depending on the respective mechanisms of the specific insults and associated tissue contexts. Therefore, throughout this study, we included STS, an apoptosis induction model used extensively in a variety of cell types, including neurons to study neurodegeneration and neuroprotection. ${ }^{30}$

Our data support that STS induces apoptosis in cultured primary human neurons. Interestingly, cotreatment with TIMP-1 or T2G mutant reduced apoptosis, preserved cell morphology, and increased cell viability in STS-treated human neurons equivalent to a well-known neurotrophic factor (BDNF). Microglia is the predominant target of HIV-1 infection in the CNS. Infected microglia produce progeny virus and cytokines, thereby activating bystander cells via cytokines and viral toxins. Consequently, HIV-1-induced neuronal apoptosis may be mediated by both viral- and glial cellderived soluble factors including proinflammatory cytokines. Various mechanisms have been demonstrated depending on the viral proteins, suggesting it is a complex process. Therefore, it is a novel finding that TIMP-1 protects neurons from a broad-spectrum cytotoxin. A better understanding of TIMP-1-mediated neuroprotection against HIV-1 will be helpful in developing new therapeutic strategies.

In addition to the well-described MMP-dependent actions, recently several studies have demonstrated that TIMPs perform a number of MMP-independent actions. Importantly, TIMP-2 promotes neuronal differentiation by inhibiting cell proliferation in an MMP-independent manner. ${ }^{31}$ Using the T2G mutant of N-TIMP-1, we demonstrate directly that inhibition of apoptosis of human neurons by TIMP-1 is in fact mediated independently of its effects on MMP activity. The dose of T2G used in these experiments $(28 \mathrm{ng} / \mathrm{ml} ; 2 \mathrm{nM})$ is slightly less than its $K_{\mathrm{i}}$ for MMP-9, ${ }^{15}$ a concentration at which it would be expected to only partially $(<50 \%)$ inhibit MMP-9. Our data show that TIMP-1-mediated neuroprotection is, at least in part, MMP-independent and an addition into its nonclassical functions because the T2G mutant was effective as 


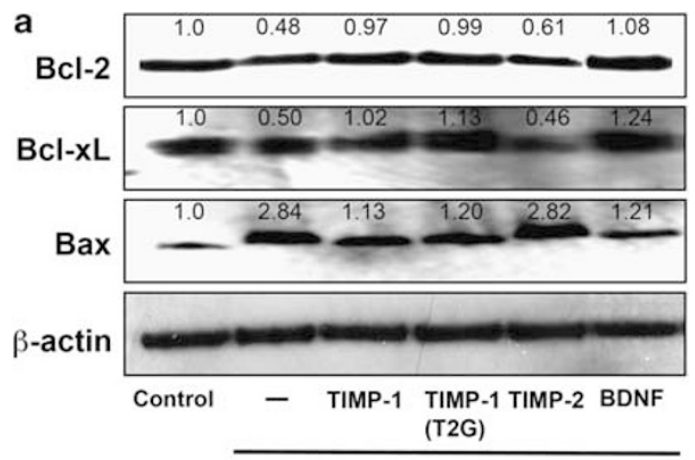

Staurosporine $(0.3 \mu \mathrm{M})$

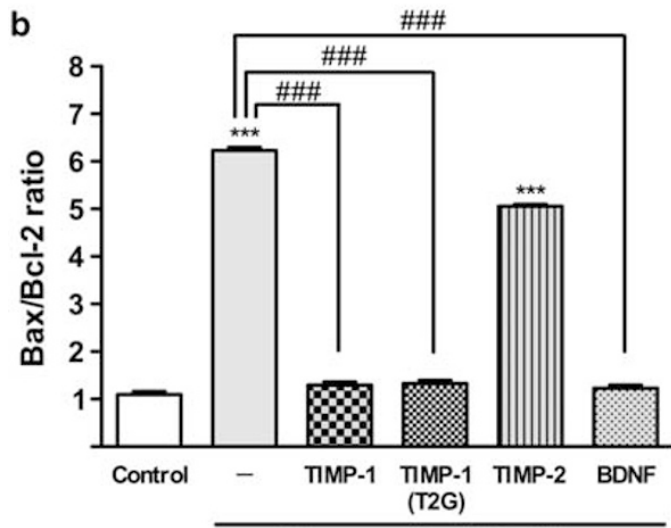

Staurosporine $(0.3 \mu \mathrm{M})$

C

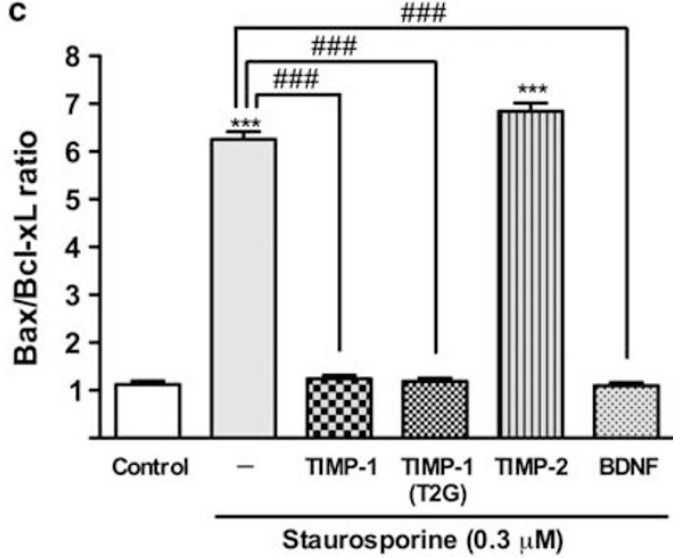

Figure 6 TIMP-1 enhances the $\mathrm{Bcl}-2$ and $\mathrm{BCl}-\mathrm{x}_{\perp}$ expression and decreases the $\mathrm{Bax} / \mathrm{Bcl}-2$ and $\mathrm{Bax} / \mathrm{Bcl}-\mathrm{x}_{\mathrm{L}}$ ratios in toxin-challenged neurons. Western blot analysis was performed on whole-cell lysates of primary human neurons challenged with STS $(0.3 \mu \mathrm{M})$ for $24 \mathrm{~h}$, with or without $50 \mathrm{ng} / \mathrm{ml}$ of TIMP-1, TIMP-2, BDNF, or $28 \mathrm{ng} / \mathrm{ml}$ T2G for the expression of $\mathrm{Bcl}-2, \mathrm{Bcl}-\mathrm{x}_{\mathrm{L}}, \mathrm{Bax}$ and $\beta$-actin. Densitometric analysis was performed and protein expression relative to $\beta$-actin in each treatment is presented on top of each band (a). The fate of cell survival/apoptosis is presented by ratios of $\mathrm{Bax} / \mathrm{Bcl}-2$ and $\mathrm{Bax} / \mathrm{Bcl}-\mathrm{x}_{\mathrm{L}}(\mathbf{b}$ and $\mathbf{c})$. Symbols centered over the error bar indicate the relative level of the significance compared with untreated control $\left({ }^{* \star *} P<0.001\right)$ or STS-alone $\left({ }^{\# \#} P<0.001\right)$

wild type TIMP-1; but other MMP-inhibitors, TIMP-2 or -3, did not protect neurons from STS-induced apoptosis. In contrast to Tan et al.'s study, ${ }^{11}$ where they demonstrated that TIMP-1-mediated neuroprotection against glutamate is
MMP-dependent; in our STS model, neuroprotection was independent of MMP-inhibition activity. This seeming disagreement with our results may be due to different paradigms, toxic mechanisms and species used in the two studies. There are significant sequence differences between the rat and human TIMP-1 most notably in the crucial N-terminal region, ${ }^{15}$ human is CTCVPPH and rat is CSCAPTH, which has large effects on specificity and might reflect differences in targets between the two species. Further, in our study we supplied TIMP-1 exogenously to neurons, which is similar to the natural method of TIMP-1 delivery, as compared with transfection of neurons. ${ }^{11}$ We utilized STS and HIV-1 $1_{\text {ADA }}$ to cause cytotoxicity. Exactly how MMP-independent effects are mediated at the molecular level is still to be determined. However, studies suggest that TIMPs can bind to a variety of cellsurface receptors, including CD63 for TIMP-1, $\alpha 3 \beta 1$ integrin for TIMP-2 and TIMP-3 to the vascular endothelial growth factor receptor-2, raising the possibility that TIMPs can directly signal through these specific receptors. ${ }^{6,32,33}$ Our study indicates a possible neurotrophic pathway by TIMP-1, independent of binding, sequestration, or inhibition of MMPs. It is possible that pro-survival pathways are activated in cells directly by TIMP-1 without involvement of MMP-binding.

Outer mitochondrial membrane permeability is regulated by $\mathrm{Bcl}-2$ family protein expression and the ratio of $\mathrm{Bax}$ to $\mathrm{Bcl}-2$. $^{34}$ Our results demonstrate that TIMP-1 attenuates STS-induced neurotoxicity through upregulation of anti-apoptotic proteins, $\mathrm{Bcl}-2$ and $\mathrm{Bcl}-\mathrm{x}_{\mathrm{L}}$, and downregulation of the pro-apoptotic protein, Bax. Data demonstrate that TIMP-1 preserves the $\mathrm{Bax} / \mathrm{Bcl}-2$ and $\mathrm{Bax} / \mathrm{Bcl}-\mathrm{x}_{\mathrm{L}}$ ratios at basal control levels. Further, this effect was direct, independent of MMP-inhibitory activity and equal to BDNF as the T2G mutant and BDNF had equivalent effects. Our data demonstrate that STS-induced neurotoxicity in human neurons involves changes in Bax/Bcl-2 or $\mathrm{Bax} / \mathrm{Bcl}-\mathrm{x}_{\mathrm{L}}$ ratios and opening of $\mathrm{MPTP}$. Our data show that TIMP-1 inhibits the opening of MPTP induced by either STS or HIV-1, and thus, indicates direct trophic signaling by TIMP-1 in human neurons.

As TIMP-1 is a small extracellular protein, any potential signaling is likely to be mediated through a cell-surface receptor. To activate pro-survival or anti-apoptotic pathways, TIMP-1 modulates $\mathrm{Bcl}-2$, Bax, and $\mathrm{Bcl}-\mathrm{x}_{\mathrm{L}}$ protein levels during neurotrophic factor deprivation and through phosphotidylinositol-3 kinase (PI3K), c-Jun N-terminal kinase, JAK2/PI3K/Akt pathways as shown in a variety of cell types. ${ }^{8,20}$ Interestingly, TIMP-1 binds to cell surface targets and translocates into the nucleus. ${ }^{35,36}$ Recently, CD63, a tetraspanin, which modulates signaling by integrin complexes, has been identified as a cellsurface interacting partner for TIMP-1 in the non-malignant breast epithelial cell line, MCF $10 \mathrm{~A}{ }^{6}{ }^{6}$ Interestingly, this study characterized the C-terminal domain of TIMP-1 as the interaction partner for CD63, whereas the N-TIMP-1 mutant T2G that lacks the $\mathrm{C}$-terminal domain, was sufficient for neuroprotection in our study. ${ }^{6,37}$ Moreover, modulation of antiapoptotic proteins by TIMP-1 in human neurons suggests that different domains of the TIMP-1 protein may mediate several separate signaling pathways. This appears to indicate that TIMP-1 neuroprotective signaling can be mediated through other receptors and/or interaction partners besides CD63 and the net outcome of these signaling pathways confers 
neuroprotection. This finding, along with the diverse antiapoptotic or growth promoting pathways activated or modulated by TIMP-1 indicates mechanisms not classically mediated through MMPs.

In summary, TIMP-1 expression by glial cells is neuroprotective against general neurotoxicity, exogenous HIV-1 and possibly from neuroinflammation or neurodegenerative diseases. Moreover, our results indicate that TIMP-1-neuroprotection could be mediated through a neurotrophic pathway without the involvement of MMPs. We also demonstrate that TIMP-1 modulates the anti-apoptotic/pro-survival pathways in human neurons. Therefore, we propose that the TIMP-1 protein is a neuroprotective signal to neurons. In this regard, the measurement of TIMP-1 level in the CSF of CNS disease patients could be a novel prognostic tool to predict the pathological outcome, while careful immune-modulation to manipulate TIMP-1 levels may slow or reverse neuronal damage in CNS diseases and injuries. Furthermore, interventions to restore or supplement TIMP-1 during the early stages of neuronal inflammation may significantly decrease the severity of neurotoxicity, tissue damage and associated degenerative symptoms. Bearing in mind the broad range of signaling activities of TIMP-1 in other cell types, it would be important to dissect the precise signaling pathways modulated by TIMP-1 that affect neuron viability before implementation of TIMP-1-delivery therapy for CNS neurodegeneration.

\begin{abstract}
Materials and Methods
Primary human neuron cultures and treatments. Human fetal neurons were isolated and cultured from first and early second trimester elective aborted specimens obtained from the Birth Defects Laboratory, University of Washington (Seattle, WA, USA) as previously described. ${ }^{38}$ The procedure was followed in full compliance with the ethical guidelines of the National Institutes of Health $(\mathrm{NIH})$, Bethesda, MD, USA; the University of Washington, the University of Nebraska, Omaha, NE, USA; and North Texas Health Science Center, Fort Worth, TX, USA. The neurons were seeded at density of $0.1 \times 10^{6} \mathrm{cells} / \mathrm{cm}^{2}$ in poly-Dlysine coated 48-well plates. Neuronal cultures were assessed after 2 weeks by immunostaining with antibodies to neuronal marker, MAP-2 (Millipore, Billerica, MA, USA) and astrocyte marker, glial fibrillary acidic protein (GFAP, Dako North America, Inc., Carpinteria, CA, USA). The culture enriched with $>90 \%$ neurons were utilized in neurotoxicity/neuroprotection experiments. STS (Merck, KGaA, Darmstadt, Germany) or HIV-1 was added with and without following proteins: TIMP-1, -2, -3, BDNF (R\&D Systems, Minneapolis, MN, USA) or T2G mutant. In experiments utilizing both TIMP-1 and T2G, the differences in molecular weights of whole TIMP-1 protein and the N-terminal domain of TIMP-1 were taken into consideration; therefore, $28 \mathrm{ng} / \mathrm{ml}$ of T2G was used alongside of $50 \mathrm{ng} / \mathrm{ml} \mathrm{TIMP-1}$ to provide the same molar concentration of proteins.
\end{abstract}

Neurotoxicity/protection assays. Various cell viability, apoptosis detection and quantitation assays were used. MTT assay, a colorimetric assay for metabolic activity measurement was performed for cell viability as described previously. ${ }^{39}$ DNA fragmentation was assayed using the dsDNA ELISA (Roche Diagnostics, Indianapolis, IN, USA) according to the manufacturer's directions. Apoptotic cells were visualized using the TUNEL assay, using In Situ Cell Death Detection Kit according to the manufacturer's directions (Roche), and counterstained with DAPI (Life Technologies). Trypan-blue exclusion assay was performed using $0.2 \%$ trypan-blue in hank's buffered salt solution (HBSS, Life Technologies) on cells for $15 \mathrm{~min}$ to observe cell membrane integrity. Live cells exclude trypan-blue staining; however, dye traverses the membrane in dead cells giving a distinctive blue color. In TUNEL assay and trypan-blue exclusion assay, microscope images were taken on an Eclipse TE-300 inverted microscope (Nikon Instruments Inc., Melville, NY, USA). For each experimental condition, a total of five to ten random fields from multiple replicates were taken at magnification X200. Microscopic images presented in this paper show representative data from at least three independent donors per experiment. Cells in microscope images were quantified as number of stained cells/total number of cells. Each micrograph contained an average of 200 cells.

MPTP assay. Opening of mPTP in cultured primary human neurons treated with STS $(0.3 \mu \mathrm{M})$ or HIV- $1_{\text {ADA }}$ with or without TIMP-1 was assessed by calcein/ $\mathrm{Co}^{2+}$-quenching technique as described by using the MitoProbe Transition Pore Assay Kit (Life Technologies) according to the manufacturer's instructions. ${ }^{40}$ Briefly, cells were loaded with $1 \mu \mathrm{M}$ calcein-AM (green) $1 \mu \mathrm{M}$ Hoechst (blue), $2 \mathrm{~mm}$ $\mathrm{CoCl}_{2}$ and $20 \mathrm{~nm}$ MitoTracker Red (Life Technologies) at $37^{\circ} \mathrm{C}$ for $15-20 \mathrm{~min}$ in phenol red free HBSS having $1.26 \mathrm{~mm} \mathrm{Ca}^{2+}$ and $0.5 \mathrm{~mm} \mathrm{Mg}^{2+}$ supplemented with $10 \mathrm{~mm}$ glutamine, $10 \mathrm{~mm}$ HEPES (Life Technologies). After washings, live cells were imaged using Nikon Eclipse TE-300 microscope with appropriate excitation and emission filters for fluorescein. The change in green fluorescence intensity and from green/yellow to red indicates mPTP opening. $\mathrm{CoCl}_{2}$ quenched all fluorescence outside the mitochondria, which facilitates the elucidation of the subtle phenomena associated with mitochondrial permeability transition leading to programmed cell death.

Immunoblot analysis. Primary human neurons were cultured as adherent monolayers in $25 \mathrm{~cm}^{2}$ flasks at a density of $2.5 \times 10^{6}$ cells per flask. The following day, cells were treated with $0.3 \mu \mathrm{M}$ STS with/without $50 \mathrm{ng} / \mathrm{ml}$ BDNF, TIMP-1, TIMP-2 ( $50 \mathrm{ng} / \mathrm{ml}$ each, R\&D Systems) or $28 \mathrm{ng} / \mathrm{ml} \mathrm{T2G}$ mutant. Whole-cell lysates were prepared after $24 \mathrm{~h}$, resolved by $12 \%$ sodium dodecyl sulfate polyacrylamide gel electrophoresis and subsequently transferred to a nitrocellulose membrane using i-Blot (Life Technologies). The membrane was blocked with 5\% BSA (Sigma, St. Louis, MO, USA) in PBST (phosphate-buffered saline, pH7.4, 0.1\% Tween-20) for $1 \mathrm{~h}$ before hybridizing with anti- $\beta$-actin $(1: 5000$, Santa Cruz Biotechnology, Santa Cruz, CA, USA), anti-Bcl-2 (1:250), anti-Bcl- $\mathrm{x}_{\mathrm{L}}(1: 250)$ or anti-Bax $(1: 200$, all from Abcam, Cambridge, MA, USA) and thereafter, in species-specific secondary antibodies (1:5000). The western blot was visualized and band intensities were quantified by densitometry analysis (Proteinsimple, Santa Clara, CA, USA).

Statistical analysis. All quantitative assays were analyzed using Prism 5.0 (GraphPad software, La Jolla, CA, USA) with one-way analysis of variance followed by Newman-Keuls multiple comparisons post-tests. Significance level was set at $P<0.05$. All experiments are expressed as mean \pm S.E.M. of triplicates and are representative of a minimum of three independent donors.

\section{Conflict of Interest}

The authors declare no conflict of interest.

Acknowledgements. This work was supported in part by R01 NS48837 from NINDS to AG. The T2G mutant utilized in these studies was supported by grant RO1 AR40994 from NIAMS, NIH to KB. The project entitled 'Laboratory of Developmental Biology' was supported by NIH Award Number 5R24HD0008836 from the Eunice Kennedy Shriver National Institute of Child Health \& Human Development. The content does not necessarily represent the official views of the Eunice Kennedy Shriver National Institute of Child Health and Human Development of the NIH. We would like to thank Ms Raisa Persidsky for her technical help in TUNEL staining.

1. Stetler-Stevenson WG. Tissue inhibitors of metalloproteinases in cell signaling: metalloproteinase-independent biological activities. Sci Signal 2008; 1: re6.

2. Kaczmarek L, Lapinska-Dzwonek J, Szymczak S. Matrix metalloproteinases in the adult brain physiology: a link between c-Fos, AP-1 and remodeling of neuronal connections? EMBO J 2002; 21: 6643-6648.

3. Crocker SJ, Whitmire JK, Frausto RF, Chertboonmuang P, Soloway PD, Whitton JL et al. Persistent macrophage/microglial activation and myelin disruption after experimental autoimmune encephalomyelitis in tissue inhibitor of metalloproteinase-1-deficient mice. Am J Pathol 2006; 169: 2104-2116.

4. Althoff GE, Wolfer DP, Timmesfeld N, Kanzler B, Schrewe H, Pagenstecher A. Long-term expression of tissue-inhibitor of matrix metalloproteinase- 1 in the murine central nervous system does not alter the morphological and behavioral phenotype but alleviates the course of experimental allergic encephalomyelitis. Am J Pathol 2010; 177: 840-853.

5. Jourquin J, Tremblay E, Bernard A, Charton G, Chaillan FA, Marchetti E et al. Tissue inhibitor of metalloproteinases-1 (TIMP-1) modulates neuronal death, axonal plasticity, and learning and memory. Eur J Neurosci 2005; 22: 2569-2578. 
6. Jung K, Liu X, Chirco R, Fridman R, Kim H. Identification of CD63 as a tissue inhibitor of metalloproteinase-1 interacting cell surface protein. EMBO J 2006; 25: 3934-3942.

7. Tsagaraki I, Tsilibary EC, Tzinia AK. TIMP-1 interaction with alphavbeta3 integrin confers resistance to human osteosarcoma cell line MG-63 against TNF-alpha-induced apoptosis. Cell Tissue Res 2010; 342: 87-96.

8. Chao C, Ghorpade A. Production and Roles of Glial Tissue Inhibitor of Metalloproteinases1 in Human Immunodeficiency Virus-1-Associated Dementia Neuroinflammation: a Review. Am J Infect Dis 2009; 5: 314-320.

9. Suryadevara R, Holter S, Borgmann K, Persidsky R, Labenz-Zink C, Persidsky Y et al. Regulation of tissue inhibitor of metalloproteinase- 1 by astrocytes: links to HIV-1 dementia Glia 2003; 44: 47-56.

10. Welser-Alves JV, Crocker SJ, Milner R. A dual role for microglia in promoting tissue inhibitor of metalloproteinase (TIMP) expression in glial cells in response to neuroinflammatory stimuli. J Neuroinflammation 2011; 8: 61.

11. Tan HK, Heywood D, Ralph GS, Bienemann A, Baker AH, Uney JB. Tissue inhibitor of metalloproteinase 1 inhibits excitotoxic cell death in neurons. Mol Cell Neurosci 2003; 22 98-106.

12. Li LW, Liu YY, Yan J, Mao YF, Luo YH, Li SP. [Apoptosis and ultrastructural lesions in Vero and J774A.1 cells induced by Leptospira interrogans]. Zhejiang Da Xue Xue Bao Yi Xue Ban 2005; 34: 4-8.

13. Seo SR, Seo JT. Calcium overload is essential for the acceleration of staurosporine induced cell death following neuronal differentiation in PC12 cells. Exp Mol Med 2009; 41 : 269-276.

14. Nguyen TL, Kim CK, Cho JH, Lee KH, Ahn JY. Neuroprotection signaling pathway of nerve growth factor and brain-derived neurotrophic factor against staurosporine induced apoptosis in hippocampal H19-7/IGF-IR [corrected]. Exp Mol Med 2010; 42: 583-595.

15. Hamze AB, Wei S, Bahudhanapati H, Kota S, Acharya KR, Brew K. Constraining specificity in the N-domain of tissue inhibitor of metalloproteinases-1; gelatinase-selective inhibitors. Protein Sci 2007; 16: 1905-1913.

16. Meng Q, Malinovskii V, Huang W, Hu Y, Chung L, Nagase H et al. Residue 2 of TIMP-1 is a major determinant of affinity and specificity for matrix metalloproteinases but effects of substitutions do not correlate with those of the corresponding P1' residue of substrate. J Biol Chem 1999; 274: 10184-10189.

17. Brew K, Nagase $H$. The tissue inhibitors of metalloproteinases (TIMPs): an ancient family with structural and functional diversity. Biochim Biophys Acta 2010; 1803: 55-71.

18. Precht TA, Phelps RA, Linseman DA, Butts BD, Le SS, Laessig TA et al. The permeability transition pore triggers Bax translocation to mitochondria during neuronal apoptosis Cell Death Differ 2005; 12: 255-265.

19. Green DR, Reed JC. Mitochondria and apoptosis. Science 1998; 281: 1309-1312.

20. Lindsten $\mathrm{T}$, Zong WX, Thompson CB. Defining the role of the Bcl-2 family of proteins in the nervous system. Neuroscientist 2005; 11: 10-15.

21. Fitzgerald JC, Ufer C, De Girolamo LA, Kuhn H, Billett EE. Monoamine oxidase-A modulates apoptotic cell death induced by staurosporine in human neuroblastoma cells. J Neurochem 2007; 103: 2189-2199.

22. Shacka JJ, Roth KA. Regulation of neuronal cell death and neurodegeneration by members of the Bcl-2 family: therapeutic implications. Curr Drug Targets CNS Neurol Disord 2005; 4: 25-39.

23. Nagase H, Visse R, Murphy G. Structure and function of matrix metalloproteinases and TIMPs. Cardiovasc Res 2006; 69: 562-573.

24. Kintscher U, Kon D, Wakino S, Goetze S, Graf K, Fleck E et al. Doxazosin inhibits monocyte chemotactic protein 1-directed migration of human monocytes. J Cardiovasc Pharmacol 2001; 37: 532-539.
25. Fujimoto M, Takagi Y, Aoki T, Hayase M, Marumo T, Gomi M et al. Tissue inhibitor of metalloproteinases protect blood-brain barrier disruption in focal cerebral ischemia. J Cereb Blood Flow Metab 2008; 28: 1674-1685.

26. Ould-yahoui A, Tremblay E, Sbai O, Ferhat L, Bernard A, Charrat E et al. A new role for TIMP-1 in modulating neurite outgrowth and morphology of cortical neurons. PLOS One 2009; 4: e8289.

27. Moore CS, Milner R, Nishiyama A, Frausto RF, Serwanski DR, Pagarigan RR et al. Astrocytic tissue inhibitor of metalloproteinase-1 (TIMP-1) promotes oligodendrocyte differentiation and enhances CNS myelination. J Neurosci 2011; 31: 6247-6254.

28. Magnoni S, Baker A, Thomson S, Jordan G, George SJ, McColl BW et al. Neuroprotective effect of adenoviral-mediated gene transfer of TIMP-1 and -2 in ischemic brain injury. Gene Ther 2007; 14: 621-625.

29. Crocker SJ, Milner R, Pham-Mitchell N, Campbell IL. Cell and agonist-specific regulation of genes for matrix metalloproteinases and their tissue inhibitors by primary glial cells. J Neurochem 2006; 98: 812-823.

30. Kabir J, Lobo M, Zachary I. Staurosporine induces endothelial cell apoptosis via focal adhesion kinase dephosphorylation and focal adhesion disassembly independent of focal adhesion kinase proteolysis. Biochem J 2002; 367(Pt 1): 145-155.

31. Perez-Martinez L, Jaworski DM. Tissue inhibitor of metalloproteinase-2 promotes neuronal differentiation by acting as an anti-mitogenic signal. J Neurosci 2005; 25: 4917-4929.

32. Seo DW, Li H, Guedez L, Wingfield PT, Diaz T, Salloum R et al. TIMP-2 mediated inhibition of angiogenesis: an MMP-independent mechanism. Cell 2003; 114: 171-180.

33. Qi JH, Ebrahem Q, Moore N, Murphy G, Claesson-Welsh L, Bond M et al. A novel function for tissue inhibitor of metalloproteinases-3 (TIMP3): inhibition of angiogenesis by blockage of VEGF binding to VEGF receptor-2. Nat Med 2003; 9: 407-415.

34. Marzo I, Brenner C, Zamzami N, Jurgensmeier JM, Susin SA, Vieira HL et al. Bax and adenine nucleotide translocator cooperate in the mitochondrial control of apoptosis. Science 1998; 281: 2027-2031.

35. Zhao WQ, Li H, Yamashita K, Guo XK, Hoshino T, Yoshida S et al. Cell cycle-associated accumulation of tissue inhibitor of metalloproteinases-1 (TIMP-1) in the nuclei of human gingival fibroblasts. J Cell Sci 1998; 111(Pt 9): 1147-1153.

36. Ritter LM, Garfield SH, Thorgeirsson UP. Tissue inhibitor of metalloproteinases-1 (TIMP-1) binds to the cell surface and translocates to the nucleus of human MCF-7 breast carcinoma cells. Biochem Biophys Res Commun 1999; 257: 494-499.

37. Huang W, Meng Q, Suzuki K, Nagase H, Brew K. Mutational study of the amino-terminal domain of human tissue inhibitor of metalloproteinases 1 (TIMP-1) locates an inhibitory region for matrix metalloproteinases. J Biol Chem 1997; 272: 22086-22091.

38. Deshpande M, Zheng J, Borgmann K, Persidsky R, Wu L, Schellpeper C et al. Role of activated astrocytes in neuronal damage: potential links to HIV-1-associated dementia. Neurotox Res 2005; 7: 183-192.

39. Manthrope M, Fagnani R, Skaper SD, Varon S. An automated colorimetric microassay for neurotrophic factors. Dev Brain Res 1986; 25: 191-198.

40. Gillessen T, Grasshoff C, Szinicz L. Mitochondrial permeability transition can be directly monitored in living neurons. Biomed Pharmacother 2002; 56: 186-193.

Cell Death and Disease is an open-access journal published by Nature Publishing Group. This work is licensed under the Creative Commons Attribution-NonCommercial-No Derivative Works 3.0 Unported License. To view a copy of this license, visit http://creativecommons.org/licenses/by-nc-nd/3.0/ 